\title{
Salidroside Protects Dopaminergic Neurons by Preserving Complex I Activity via DJ-1/Nrf2-Mediated Antioxidant Pathway
}

\author{
Ruru Li $\mathbb{D},{ }^{1}$ Songhai Wang, ${ }^{1}$ Tao Li $\left(\mathbb{D},{ }^{1}\right.$ Leitao $W u,{ }^{1}$ Yan Fang, ${ }^{1}$ Yang Feng $\mathbb{D}^{1},{ }^{1}$ \\ Lingling Zhang $\mathbb{D},{ }^{1}$ Jianzong Chen $\mathbb{D}^{1},{ }^{1}$ and Xin Wang $\mathbb{D}^{2}$ \\ ${ }^{1}$ Department of Chinese Medicine, Xijing Hospital, Fourth Military Medical University, Xi'an 710032, China \\ ${ }^{2}$ Department of Toxicology, Faculty of Preventive Medicine, Fourth Military Medical University, Xi'an 710032, China \\ Correspondence should be addressed to Jianzong Chen; jzchen57@fmmu.edu.cn and Xin Wang; xinwang@fmmu.edu.cn
}

Received 14 January 2019; Revised 9 March 2019; Accepted 1 April 2019; Published 16 May 2019

Academic Editor: Jan Aasly

Copyright (C) 2019 Ruru Li et al. This is an open access article distributed under the Creative Commons Attribution License, which permits unrestricted use, distribution, and reproduction in any medium, provided the original work is properly cited.

\begin{abstract}
The pathogenic mechanism of Parkinson's disease (PD) remains to be elucidated; however, mitochondrial dysfunction at the level of complex I and oxidative stress is suggestively involved in the development of PD. In our previous work, salidroside (Sal), an active component extracted from the medicinal plant Rhodiola rosea L., might protect dopaminergic (DA) neurons through modulating ROS-NO-related pathway. However, the mechanism of Sal-induced neuroprotective effects against PD remains poorly understood. Therefore, we further investigated whether Sal plays neuroprotective effects by activating complex I via DJ-1/Nrf2-mediated antioxidant pathway. The results showed that Sal remarkably attenuated $\mathrm{MPP}^{+} / \mathrm{MPTP}^{-i n d u c e d}$ decline in cell viability, accompanied by decreases in reactive oxygen species (ROS), malondialdehyde (MDA), and 8-hydroxydeoxyguanosine (8-OHdG) contents and increases in the superoxide dismutase (SOD), catalase (CAT), and glutathione peroxidase (GSH-Px), as well as glutathione (GSH) levels. Furthermore, Sal greatly improved the behavioral performance and prevented the severe reduction of TH-positive neuron numbers in the substantia nigra (SN). Moreover, in comparison with the $\mathrm{MPP}^{+} / \mathrm{MPTP}$ group, Sal increased the nuclear translocation of DJ-1 and Nrf2 and the mitochondrial translocation of DJ-1, accompanied by activating complex I. Furthermore, silencing of DJ-1/Nrf2 inhibited the increase of complex I activity and cell viability elicited by Sal. Together, these results support the neuroprotective effect of Sal against $\mathrm{MPP}^{+} / \mathrm{MPTP}^{-i n d u c e d} \mathrm{DA}$ neurons damage.
\end{abstract}

\section{Introduction}

Parkinson's disease (PD) is a prevalent neurodegenerative movement disorder affecting $2 \%-3 \%$ of the population above the age of 65 worldwide $[1,2]$. The hallmark of PD is the degeneration of dopaminergic (DA) neurons in the substantia nigra (SN) and the presence of Lewy bodies (LBs) [3]. The etiology of PD remains unclear, but extensive evidences from postmortem brain tissues have suggested that oxidative stress and deficiency of complex I activity are related to the pathogenic mechanism of PD $[4,5]$.

Oxidative stress, which occurs mainly due to overproduction of reactive oxygen species (ROS), activates a cascade of events leading to DA neuron degeneration [6]. Mitochondrial complex I is the major site where ROS generate, and once damaged, the mitochondria complex I would not only produce more ROS but also become the target for oxidative stress damage [7]. Complex I dysfunction can result in excess generation of ROS, which in turn will lead to the oxidative modification of complex I in a positive feedback loop to exacerbate complex I dysfunction [8]. Given the role of complex I in generating ROS and undergoing oxidative modification damage, it is a promising target for antioxidant therapeutic strategies.

Recently, researchers have focused on the pharmacological targeting of antioxidant gene transcription, including DJ-1 (gene: PARK7) and nuclear factor erythroid-2-related factor 2 (protein: Nrf2; gene: NFE2L2), attempting to inhibit oxidative stress and deactivation of complex I and limit neuronal damage $[9,10]$. DJ-1 is known to act as an 
antioxidant via directly scavenging ROS and upregulating the expression of other antioxidant genes [11]. One of the most relevant target genes is the transcriptional factor Nrf2, a master regulator of antioxidant gene, which activates a range of antioxidant enzymes via antioxidant response element (ARE) enhancer [11].

Rhodiola rosea $\mathrm{L}$. has been used for centuries in traditional medicinal practices in Asia, Scandinavia, and Eastern Europe to stimulate nerves, enhance physical and mental performance, improve resistance to high-altitude sickness, and treat fatigue, psychological stress, and depression [12, 13]. Salidroside (Sal), an active component extracted from Rhodiola rosea L., possesses multiple pharmacological properties, including antioxidant, antiaging, and antifatigue [12].

Our previous study has shown Sal plays neuroprotective effect through modulating the ROS-NO-related pathway [14]. However, the effects of Sal on complex I dysfunction, accompanied by neuronal loss induced by oxidative stress, and the protective mechanism of Sal against MPTP/MPP ${ }^{+}$ remains unclear. In light of these findings, the present study was designed to elucidate whether Sal plays neuroprotective effect through activating complex I via DJ-1/Nrf2 -elated antioxidant pathway in the $\mathrm{MPTP} / \mathrm{MPP}^{+}$-induced $\mathrm{PD}$ model.

\section{Materials and Methods}

2.1. Cell Culture and Treatment. MN9D cells, a DA neuronal cell line derived from mouse mesencephalon, were purchased from Type Culture Collection of the Chinese Academy of Sciences (Shanghai, China). The cells were cultured in RPMI 1640 medium (Hyclone, Logan, USA) with $10 \%$ FBS (Gibco, Gaithersburg, MD) and maintained at $37^{\circ} \mathrm{C}$ in a $5 \% \mathrm{CO}_{2}$ atmosphere. The cells were pretreated with Sal $(10,25$, and $50 \mu \mathrm{M})$ for $24 \mathrm{~h}$ and incubated with $200 \mu \mathrm{M}$ $\mathrm{MPP}^{+}$for an additional $24 \mathrm{~h}$. The cells were divided into the following groups: control group, $\mathrm{MPP}^{+}$group, Sal group, and groups of different concentrations of Sal before $\mathrm{MPP}^{+}$ treatment.

2.2. Cell Transfection. siRNA duplexes were synthesized and purified by Biomics Biotechnologies (Nantong, China). SiRNA was diluted to $50 \mathrm{~nm}$ with Opti-MEM. The siRNA sequences of NFE2L2 and PARK7 are depicted in Table 1. The transfection was performed using Lipofectamine 2000 (Thermo Fisher, Waltham, MA, USA). After $72 \mathrm{~h}$, the transfection efficiency was detected by western blot.

2.3. Cell Viability. Cell viability was measured using MTT assay kit (Sigma, MO, USA), following the manufacturer's instructions. The absorbance at $570 \mathrm{~nm}$ was measured, and cell viability was expressed as the percentage to the control group.

2.4. Animals and Drug Treatments. Adult male C57BL/6 mice (6-8 weeks) weighing $22-25 \mathrm{~g}$ were treated and maintained according to the guidelines established by the National Institutes of Health for the care and use of laboratory animals and were approved by the Animal Care Committee of the Fourth Military Medical University and Chinese Academy of Medical Sciences. Mice were randomly assigned to six groups (10 mice per group) including Group A, control mice; Group B, MPTP challenged; Group C, MPTP challenged and then Sal treated $(15 \mathrm{mg} / \mathrm{kg})$; Group D, MPTP challenged and then Sal treated $(50 \mathrm{mg} / \mathrm{kg}$ ); Group E, Sal treated alone $(15 \mathrm{mg} / \mathrm{kg})$; and Group F, Sal treated alone $(50 \mathrm{mg} / \mathrm{kg})$. Mice received intraperitoneal injection with MPTP for 5 consecutive days ( $30 \mathrm{mg} / \mathrm{kg} /$ day). Control mice and $\mathrm{Sal}$ alone-treated mice received intraperitoneal injection of saline for 5 days. After MPTP injection, the mice of the last four groups received intraperitoneal injection of Sal for 7 days, and the control and MPTP groups received an equivalent volume of saline. Seven days after the last administration of MPTP or saline, mice were subjected to behavioral tests.

2.5. Behavioral Tests. The behavior performance was analyzed by the pole and open field tests as previously described [15]. In brief, the pole test was performed to detect the impairment of limb movement [16]. After placing the mice on a rough pole, the time for the mice to turn completely downwards (T-turn) and climb down to the floor (T-LA) was recorded. Open field is a popular model for assessing spontaneous movement activity and anxiety-like behavior [17-19]. The mice were placed in a wooden box $\left(40 \times 40 \mathrm{~cm}^{2}\right)$ that was enclosed by $10 \mathrm{~cm}$ high walls. The brown floor with a pattern of 16 squares was marked with black lines. Animals were placed in the rear left square and allowed to freely explore the field for $5 \mathrm{~min}$. The speed was recorded to measure the locomotion level.

2.6. Measurement of ROS. The levels of ROS were measured by Reactive Oxygen Species Assay Kit (Beyotime, Beijing, China) as previously described [14]. And, the fluorescence intensities were analyzed using a microplate reader. The measured fluorescence values were expressed as a percentage of the fluorescence of the control group.

2.7. Measurement of Complex I Activity. The complex I activity was measured by the Complex I Enzyme Activity Microplate Assay Kit (Abcam, MA, USA), following the manufacturer's instructions, and OD values at $450 \mathrm{~nm}$ was recorded in the kinetic mode at room temperature (RT) for $30 \mathrm{~min}$. The complex I activity was expressed as the change in absorbance per minute per amount of sample loaded into the well.

2.8. Immunofluorescent Assay. For immunofluorescent assay, the SN of brains were isolated, frozen, and cut into $30 \mu \mathrm{m}$ slices on a Cryostats (Thermo Fisher, Waltham, MA, USA). Then, the slides were permeabilized and blocked in $2 \%$ BSA, incubated with TH antibody (Sigma-Aldrich, USA) overnight, and treated with Cy3-labeled goat anti-chicken 
TABLE 1: siRNA sequences of MN9D cells.

\begin{tabular}{lcc}
\hline Gene & Forward primer & Reverse primer $^{\prime}$ \\
\hline NFE2L2 & $5^{\prime}$-GCAAGUUUGGCAGGAGCUAdTdT-3' & $5^{\prime}$-UAGCUCCUGCCAAACUUGCdTdT-3' $^{\prime}$ \\
NFE2L2 & $5^{\prime}$-CCGAAUUACAGUGUCUUAAdTdT-3' & $5^{\prime}$-CCGAAUUACAGUGUCUUAAdTdT-3' \\
NFE2L2 & $5^{\prime}$-GCAACUGUGGUCCACAUUUdTdT-3' & $5^{\prime}$-AAAUGUGGACCACAGUUGCdTdT-3' \\
PARK7 & $5^{\prime}$-GCAGUGUAGCCGUGAUGUAdTdT-3' & $5^{\prime}$-UACAUCACGGCUACACUGCdTdT-3' \\
PARK7 & $5^{\prime}$-CAGUGUAGCCGUGAUGUAAdTdT-3' & $5^{\prime}$-UUACAUCACGGCUACACUGdTd-3' \\
\hline
\end{tabular}

IgG for $1 \mathrm{~h}$ in the dark. Images were analyzed by using an inverted microscope (IX51-12PH, Olympus) at a magnification of $200 \times$.

2.9. Western Blot. The total protein was lysed in RIPA lysis, the nuclear and cytoplasmic proteins were extracted with the Nuclear and Cytoplasmic Protein Extraction Kit, and the mitochondrial protein in the cell or SN was extracted with the Cell Mitochondria Isolation Kit or Tissue Mitochondria Isolation Kit (Beyotime, Beijing, China). Equal quantity of proteins were electrophoresed on SDS-PAGE gel and transferred to PVDF membranes. The membranes were blocked and incubated with SOD1 (CST, USA), SOD2 (CST), GSH-Px (CST), DJ-1(CST), Nrf2 (Sigma-Aldrich), and Keap1 (Sigma-Aldrich) antibodies at $4^{\circ} \mathrm{C}$ overnight followed by goat anti-rabbit IgG antibody (Santa Cruz Biotechnology). The membrane was visualized using chemiluminescent reagents, and the protein level was quantified by using Image J. All protein levels were adjusted for the corresponding $\beta$-actin (CST), histone H3 (CST), and VDAC1 (CST) and were consistent across different treatment conditions.

2.10. Measurement of MDA, 8-OHdG, GSH, GSH-Px, Cu/ZnSOD (SOD1), Mn-SOD (SOD2), and CAT in SN. To extract the protein, SN was homogenized in Tris buffer $(10 \mathrm{mM}$ Tris- $\mathrm{HCl}, 1 \mathrm{mM}$ EDTA, $0.32 \mathrm{M}$ sucrose, and $\mathrm{pH} 7.8)$ as $10 \%(\mathrm{w} / \mathrm{v})$ using a Teflon homogenizer (Glas-Col homogenizer system, Vernon Hills, USA), centrifuged at $20,000 \times \mathrm{g}$ for $10 \mathrm{~min}$. The MDA, GSH, GSH-Px, SOD1, SOD2, and CAT contents in the SN were measured by using a commercially available kit (Jiancheng Bioengineering Institute, Nanjing, China) following the manufacturer's instructions. The $8-\mathrm{OHdG}$ level was measured using 8-hydroxy 2-deoxyguanosine ELISA kit (Abcam, MA, USA), according to the manufacturer's protocol. The OD values were measured using a microplate reader and were expressed as percentage of the OD values of the control group.

2.11. Statistical Analysis. Quantitative data were reported as mean \pm standard deviation of three independent experiments. Statistical analysis among different groups was performed with one-way analysis of variance (ANOVA), followed by Tukey's multiple comparison test as post hoc. A value of $P<0.05$ was considered significant.

\section{Results}

3.1. Effect of Sal on MPP ${ }^{+}$-Induced Reduction of Cell Viability. MN9D cells were treated with various concentrations of $\mathrm{MPP}^{+}$for $24 \mathrm{~h}$. Results of MTT indicated that $200 \mu \mathrm{M}$ of $\mathrm{MPP}^{+}$remarkably reduced the cell viability (Figure $1(\mathrm{a})$ ). To examine whether Sal alone had an effect on cell viability, we treated cells with different concentrations of Sal for $24 \mathrm{~h}$. The Sal at $0-50 \mu \mathrm{M}$ had no obvious effect on cell viability (Figure 1(b)). Then, we investigated the neuroprotective effect of Sal on $\mathrm{MPP}^{+}$-induced cell toxicity, which was markedly prevented by Sal $(10,25$, and $50 \mu \mathrm{M})$ (Figure $1(\mathrm{c})$ ). Given the results of MTT, cells were pretreated with or without Sal $(10,25,50 \mu \mathrm{M})$ for $24 \mathrm{~h}$ followed by $\mathrm{MPP}^{+}$ $(200 \mu \mathrm{M})$ for an additional $24 \mathrm{~h}$ in the following experiments.

\subsection{Effect of Sal on MPTP-Induced Behavioral Deficits.} The results of the pole test and open field test are shown in Figure 2. In the pole test, the time to T-turn and T-LA is longer in MPTP-treated mice, compared with the control mice (Figures 2(a) and 2(b)). Next, in the open field test, the MPTP-treated mice were significantly slow in velocity of movement compared with the control mice $(P<0.01$; Figure 2(c)). Sal treatment significantly alleviated these behavior disorders induced by MPTP $(P<0.05$ or $P<0.01)$, and Sal alone had no apparent effect.

3.3. Effect of Sal on MPTP-Induced Reduction of TH-Positive Neurons. The results of immunofluorescent assay displayed that, in comparison with the control group, the number of TH-positive neurons in the SN of the MPTP group was significantly reduced $(P<0.01$; Figures $3(\mathrm{a})$ and $3(\mathrm{~b}))$. The number of $\mathrm{TH}$-positive neurons in the mice that were treated with 15 and $50 \mathrm{mg} / \mathrm{kg}$ Sal was significantly increased in comparison with that in the MPTP group $(P<0.05$; $P<0.01)$. Moreover, no differences in TH-positive neurons were detected in Sal alone-treated mice.

3.4. Effects of Sal on MPP $P^{+} / M P T P$-Induced Oxidative Stress. To confirm whether Sal modulated the oxidative stress, we measured the ROS, MDA, and 8-OHdG levels. As shown in Figure $4(\mathrm{a}), \mathrm{MPP}^{+}$treatment can significantly increase the ROS levels in cells, compared with those of the control group, but pretreatment with different concentrations of Sal can abolish $\mathrm{MPP}^{+}$-induced overproduction of ROS $(P<0.05, P<0.01$; Figure $4(\mathrm{a}))$. Then, Sal modulation on 


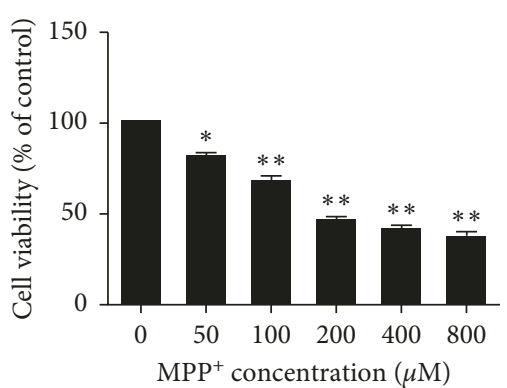

(a)

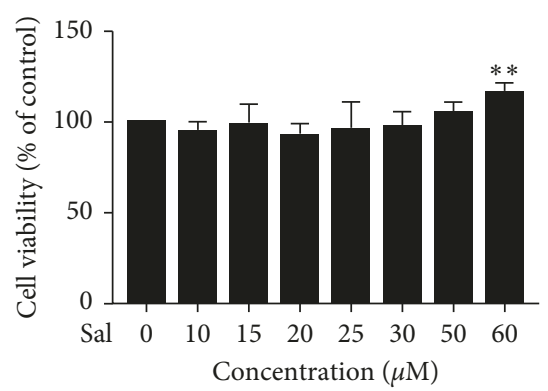

(b)

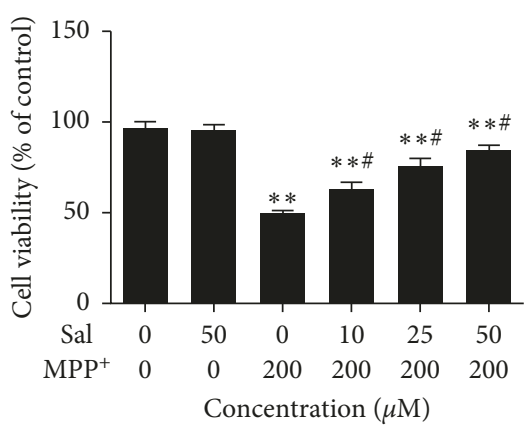

(c)

Figure 1: Sal ameliorated the cytotoxicity induced by $\mathrm{MPP}^{+}$in MN9D cells. (a) The cytotoxicity of various concentrations of MPP ${ }^{+}$on MN9D cells for $24 \mathrm{~h}$. (b) The effect of various concentrations of Sal on the cell viability for $24 \mathrm{~h}$. (c) The effect of pretreatment with Sal (10, 25, and $50 \mu \mathrm{M})$ on $\mathrm{MPP}^{+}$-induced cytotoxicity. Each column represents the mean $\pm \mathrm{SD}(n=3) .{ }^{*} P<0.05$ and ${ }^{* *} P<0.01$, compared with the control group; ${ }^{\#} P<0.05$ and ${ }^{\# \#} P<0.01$, compared with the $M P P^{+}$-treated group.

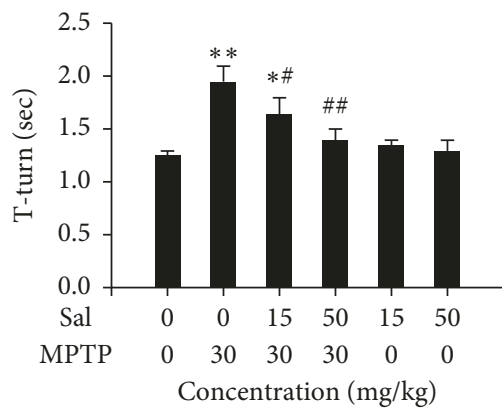

(a)

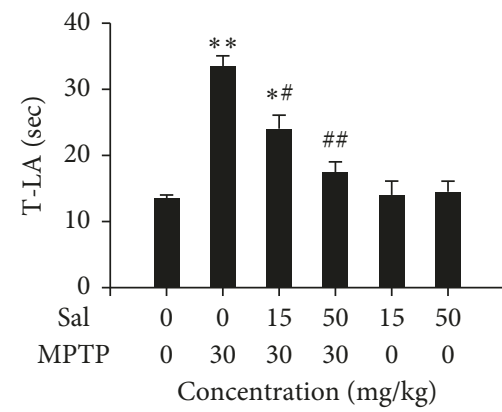

(b)

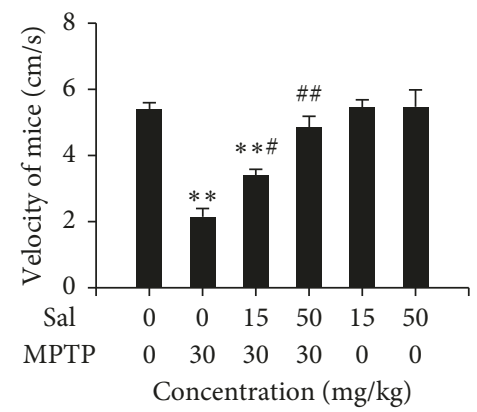

(c)

Figure 2: Effect of treatment with Sal $(15,50 \mathrm{mg} / \mathrm{kg} /$ day, 7 days), MPTP (30 mg/kg/day, 5 days), or their combination on behavior performance of mice. (a) The time obtained for mice to turn completely downward (T-turn). (b) The time obtained for mice to climb to the floor (T-LA). (c) The speed of mice in the wooden box. Each column represents the mean \pm SD $(n=10){ }^{*} P<0.05$ and ${ }^{* *} P<0.01$, compared with the control group; ${ }^{\#} P<0.05$ and ${ }^{\# \#} P<0.01$, compared with the MPTP-treated group.
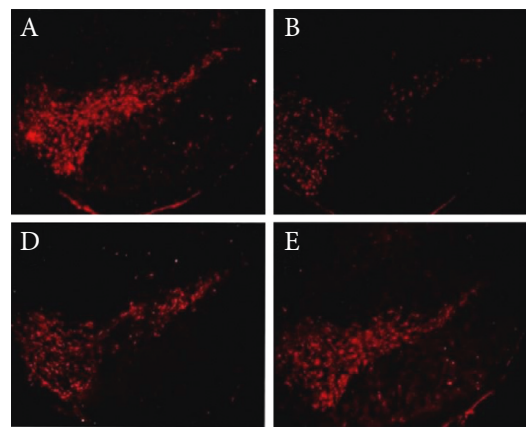

(a)

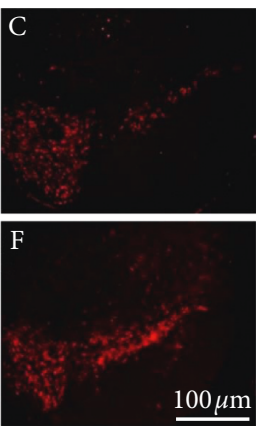

$00 \mu \mathrm{m}$

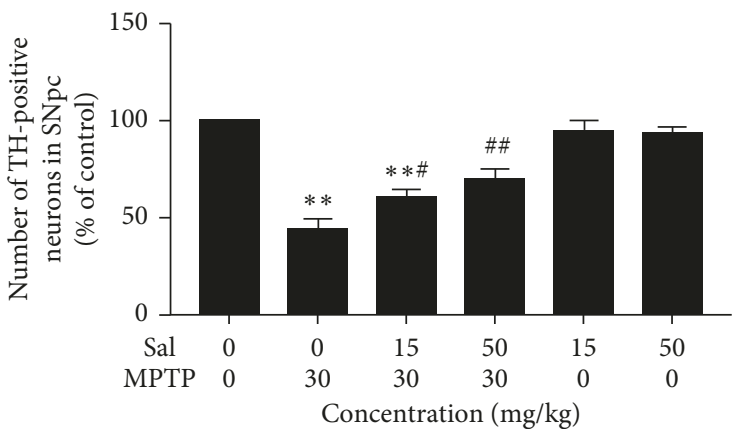

(b)

Figure 3: Effect of treatment with Sal, MPTP, or their combination on TH-positive neurons in the substantia nigra of mice. (a) Representative photomicrographs of TH-positive neurons in the substantia nigra. (b) Quantitative analysis of the number of TH-positive neurons in the substantia nigra. Each column represents the mean $\pm \mathrm{SD}(n=3) .{ }^{*} P<0.05$ and ${ }^{* *} P<0.01$, compared with the control group; ${ }^{\#} P<0.05$ and ${ }^{\# \#} P<0.01$, compared with the MPTP-treated group.

MDA and 8-OHdG levels in the SN was investigated. As shown in Figure 4(b), the MDA and 8-OHdG levels in MPTP-treated mice significantly increased, compared with the control group $(P<0.01)$. However, the accumulation of
MDA and 8-OHdG significantly reduced dose-dependently by Sal treatment $(P<0.05, P<0.01$; Figure $4(\mathrm{~b}))$. Additionally, no differences were detected in Sal alone-treated mice. 


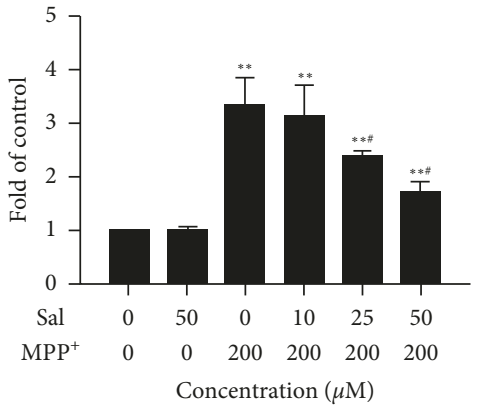

(a)

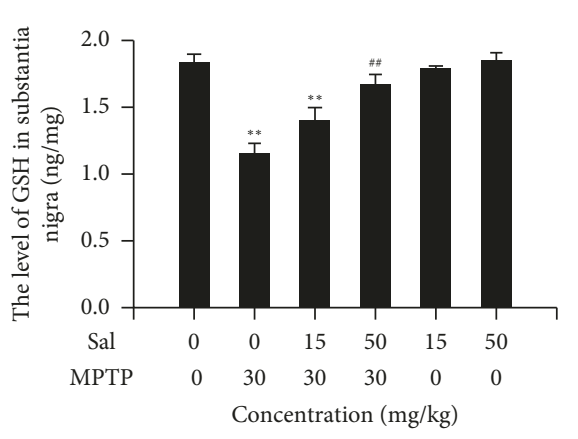

(c)

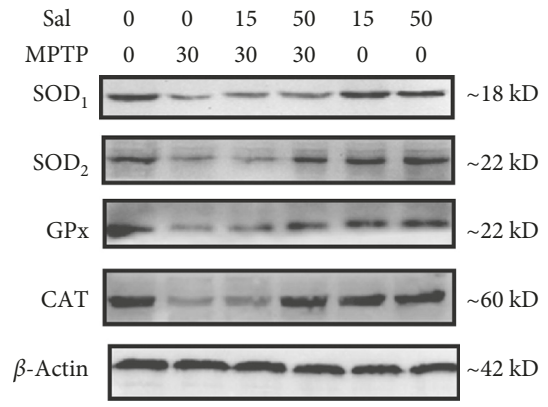

(e)

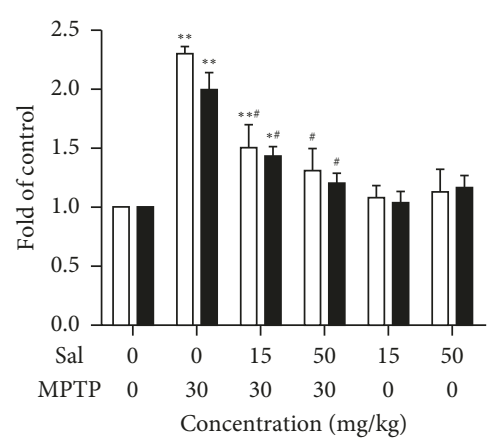

$\square \mathrm{MDA}$

- 8-OHdG

(b)

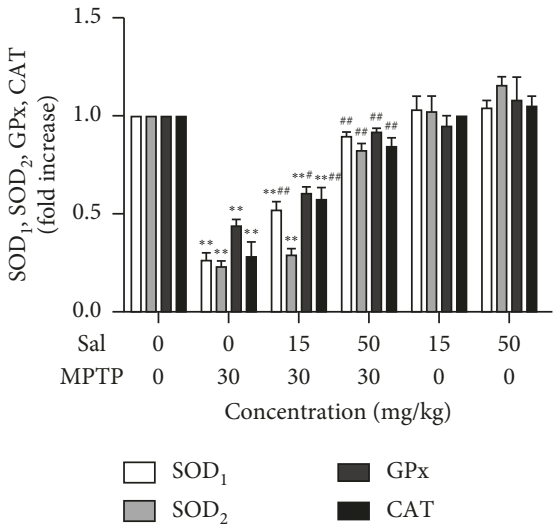

(d)

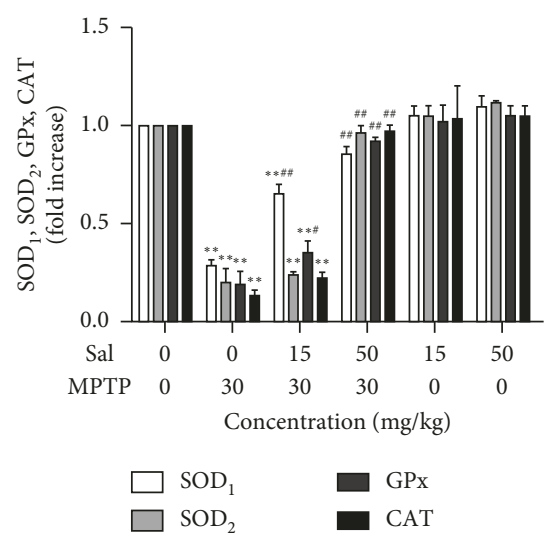

(f)

FIgURE 4: Sal inhibited oxidative stress and increased the generation of endogenous antioxidants. (a) The level of ROS in MN9D cells. (b) The level of MDA and 8-OHdG in the substantia nigra of mice. (c) The level of GSH in the substantia nigra of mice. The level (d) and the protein expression (e, f) of SOD1, SOD2, GSH-Px, and CAT in the substantia nigra of mice. Each column represents the mean \pm SD $(n=4)$. ${ }^{*} P<0.05$ and ${ }^{* *} P<0.01$, compared with the control group; ${ }^{\#} P<0.05$ and ${ }^{\# \#} P<0.01$, compared with the MPP $/ \mathrm{MPTP}^{+}$-treated group.

3.5. Effect of Sal on the Levels of Endogenous Antioxidants. In the body, oxidative stress could be counteracted by endogenous and exogenous antioxidants [20]. We investigated whether Sal can enhance the content of endogenous antioxidants by measuring the GSH, SOD1, SOD2, GSH-Px, and CAT levels, as assayed by ELISA. The GSH content in the SN of MPTP-treated group significantly decreased, compared with the control group $(P<0.01)$. However, Sal treatment significantly alleviated MPTP-induced loss of GSH (Figure 4(c)). In addition, the SOD1, SOD2, GSH-Px, and CAT activities in MPTP-treated mice significantly decreased, compared with the control group $(P<0.01)$, and Sal 
attenuated this decrease dose-dependently. Sal alone did not show a significant effect on GSH, SOD1, SOD2, GSH-Px, and CAT levels, compared with the control group (Figure 4(d)).

Western blot revealed that the expression of SOD1, SOD2, GSH-Px, and CAT was significantly decreased by MPTP treatment, compared with the control group $(P<0.01$; Figures 4(e) and 4(f)). Meanwhile, Sal treatment significantly inhibited the decrease in the expression of SOD1, SOD2, GSH-Px, and CAT dose-dependently. However, no differences were detected in Sal alone-treated mice.

3.6. Effect of Sal on the DJ-1/Nrf2 Expression In Vitro and In Vivo. To determine the contribution of DJ-1/Nrf2 on the antioxidative stress effect of Sal, we first measured the protein expression of DJ-1 and Nrf2. As shown in Figure 5(a), Sal treatment increased the nuclear and mitochondrial accumulation of DJ-1 in comparison with the $\mathrm{MPP}^{+}$group. Western blot results in vivo showed Sal treatment increased the nuclear and mitochondrial accumulation of DJ-1 in comparison with the MPTP group and no differences were detected in the Sal alone group (Figure 5(c)). Moreover, as shown in Figure 5(b), Sal treatment increased the nuclear Nrf2 and decreased the cytoplasmic expression of Keap1 in comparison with the $\mathrm{MPP}^{+}$group. However, Sal alone had no obvious influence on Nrf2 and Keap1 expression. Western blot in vivo revealed that Sal increased the nuclear Nrf2 and decreased the cytoplasmic expression of Keap 1 dose-dependently compared with MPTP-treated mice $(P<0.01, P<0.05$; Figure $5(\mathrm{~d}))$. However, transfected with DJ-1/Nrf2 siRNA abrogated the inhibitory effect of Sal on ROS generation (Figure S2) and inhibited Sal-induced nuclear/mitochondrial accumulation of DJ-1 and the nuclear accumulation of Nrf2 (Figure S3). These results supported the role of DJ-1/Nrf2 on the antioxidative stress effect of Sal.

3.7. Effects of Salon the Complex I Activity in Vitro and in Vivo. To understand the mechanisms of the protective effects of Sal in mitochondria, we measured the complex I activities in vitro and in vivo. The complex I activity of the $\mathrm{MPP}^{+}$group significantly decreased, compared with that of the control group in MN9D cells $(P<0.01$; Figure 6(a)). Moreover, pretreatment with different concentrations of Sal can abolish $\mathrm{MPP}^{+}$-induced inhibition of complex I activity dosedependently in MN9D cells. In vivo, complex I activity of the MPTP-treated group significantly decreased, compared with that of the control group $(P<0.01$; Figure $6(\mathrm{~b}))$. However, Sal treatment significantly reversed MPTPinduced decrease in complex I activity dose-dependently. Cells and mice treated by Sal alone had no significant difference compared with the control group in vitro and in vivo.

3.8. Role of DJ-1/Nrf2-Mediated Antioxidant Effect for the Maintenance of Complex I Activity and Neuroprotective Effect of Sal. To further study whether DJ-1/Nrf2-mediated antioxidant effect contributes to the maintenance of complex I activity and the neuroprotective effects of Sal, DJ-1 and Nrf2 were silenced using siRNAs (Figure S1). Data shown in Figures 6(c) and 6(d) showed that silencing of DJ-1 and Nrf2 inhibited the increase of complex I activity and cell viability elicited by Sal. These results notably indicated DJ-1/Nrf2mediated antioxidant effects plays an essential role in the neuroprotective effects of Sal.

\section{Discussion}

In the present study, we demonstrated that Sal plays a neuroprotective effect against $\mathrm{MPP}^{+} / \mathrm{MPTP}$-induced impairment of DA neurons by preserving complex I activity via DJ-1/Nrf2-mediated antioxidant pathway, as evidenced by the following: (1) Sal pretreatment significantly increased the cell viability in MN9D cells subjected to $\mathrm{MPP}^{+}$; (2) administration of Sal notably attenuated behavioral impairments, accompanied by decreased $\mathrm{TH}$-positive neurons in the SN of the MPTP-induced PD model; (3) Sal decreased the oxidative stress and increased the levels of endogenous antioxidants; (4) Sal increased the nuclear translocation of DJ-1 and Nrf2 and the mitochondrial translocation of DJ-1, accompanied by activating complex I; (5) knockdown of DJ1/Nrf2 expression with siRNA abolished the protective effects of Sal on complex I and cell viability in the $\mathrm{MPP}^{+}$induced PD model.

Oxidative stress is characterized by ROS overproduction and enzymatic and nonenzymatic antioxidant deficiency in the biological system [21]. Excessive ROS can damage all macromolecules, including nucleic acids, lipids, and proteins, leading to an overall progressive decline in physiological function [5]. In our study, we examined the oxidative stress via measuring the ROS production and levels of the oxidative products, including lipid peroxidation product (MDA) and DNA oxidation product (8-OHdG) $[22,23]$. The results indicated that Sal plays important roles in inhibiting oxidative stress, as evidenced by diminishing ROS accumulation, MDA, and 8-OHdG levels against $\mathrm{MPP}^{+} / \mathrm{MPTP}$. The antioxidant systems include enzymatic scavengers, such as SOD, CAT, and GSH-Px [20]. SODs belong to the family of metalloenzymes and can scavenge highly toxic $\mathrm{OH}^{-}$by catalyzing the dismutation of superoxide radicals into $\mathrm{O}_{2}$ and $\mathrm{H}_{2} \mathrm{O}_{2}$ [24]. Among the superfamily of SODs, SOD1 and SOD2 play important roles in eliminating excess ROS to protect tissues from oxidative damage [25]. SOD1 is mainly located in the cytoplasm and is one of the most important free-radical scavengers that respond to oxidative stress [26]. SOD2 is a mitochondrial antioxidant whose deficiency causes devastating effects to mitochondrial metabolism $[26,27]$. In addition, CAT and GSH-Px convert $\mathrm{H}_{2} \mathrm{O}_{2}$ to $\mathrm{H}_{2} \mathrm{O}$ [20]. In our present study, the activities of GSH, SOD1, SOD2, CAT, and GSH-Px were declined in MPTP-treated mice and Sal treatment ameliorated the decline in GSH, SOD1, SOD2, CAT, and GSH-Px activities. Altogether, our findings indicated the neuroprotective effect of Sal on the $\mathrm{MPP}^{+} / \mathrm{MPTP}$-induced PD model, which was associated with decreased oxidative damage and increased antioxidants.

DJ-1 is a recessively inherited Parkinson's gene and functions as an antioxidant through a variety of 

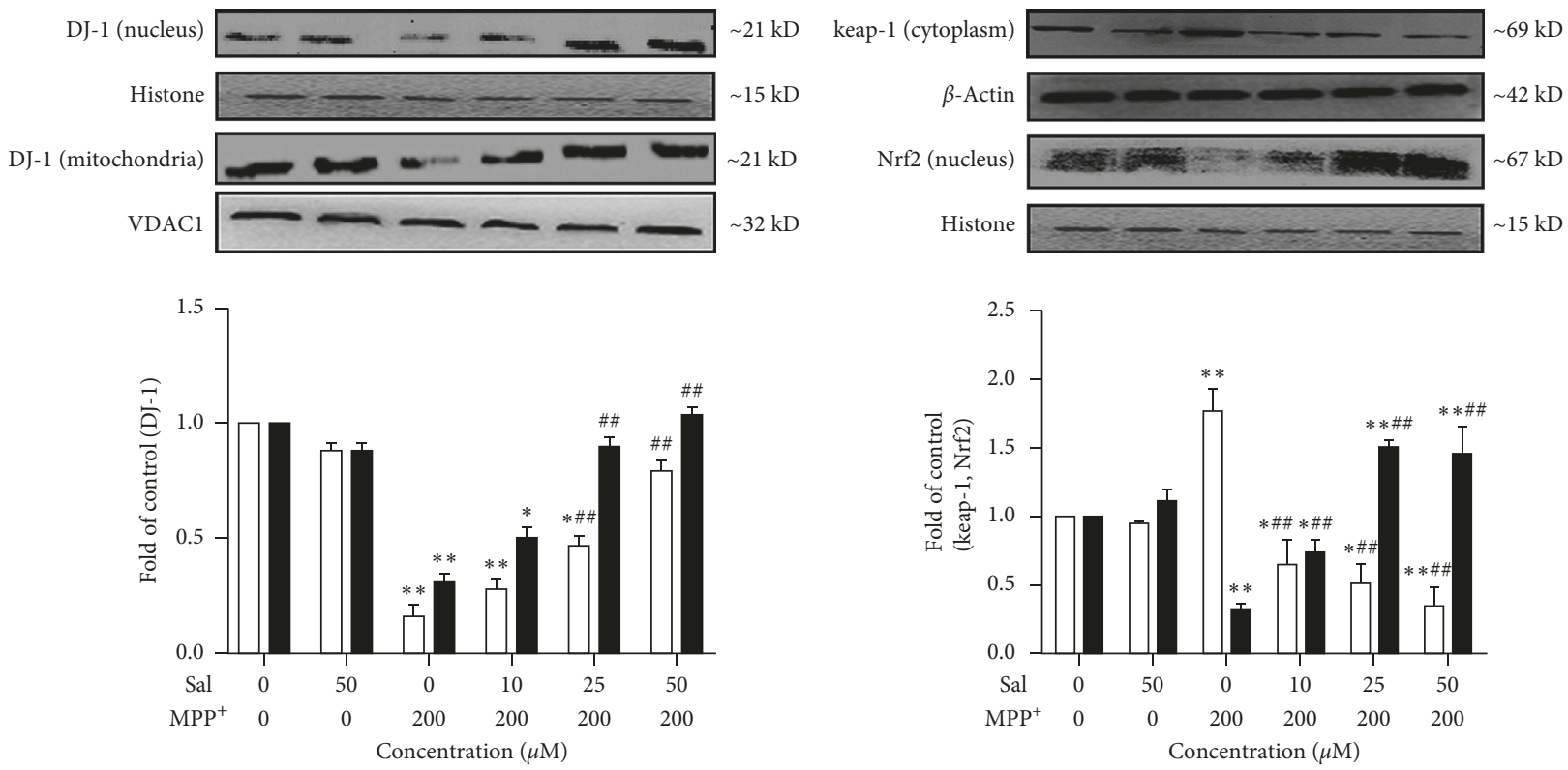

$\square$ DJ-1 in nucleus

- DJ-1 in mitochondria

(a)

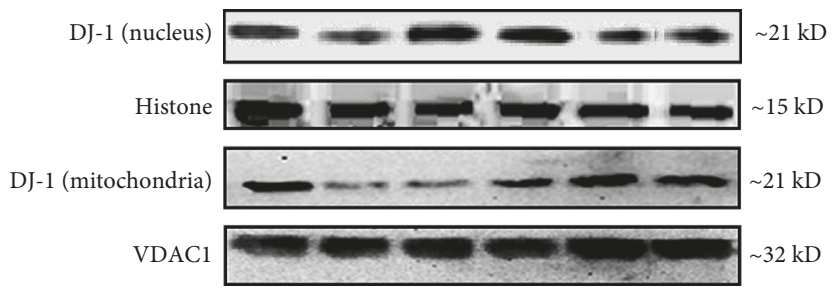

$\square$ Keap-1 in cytoplasm

- Nrf2 in nucleus

(b)
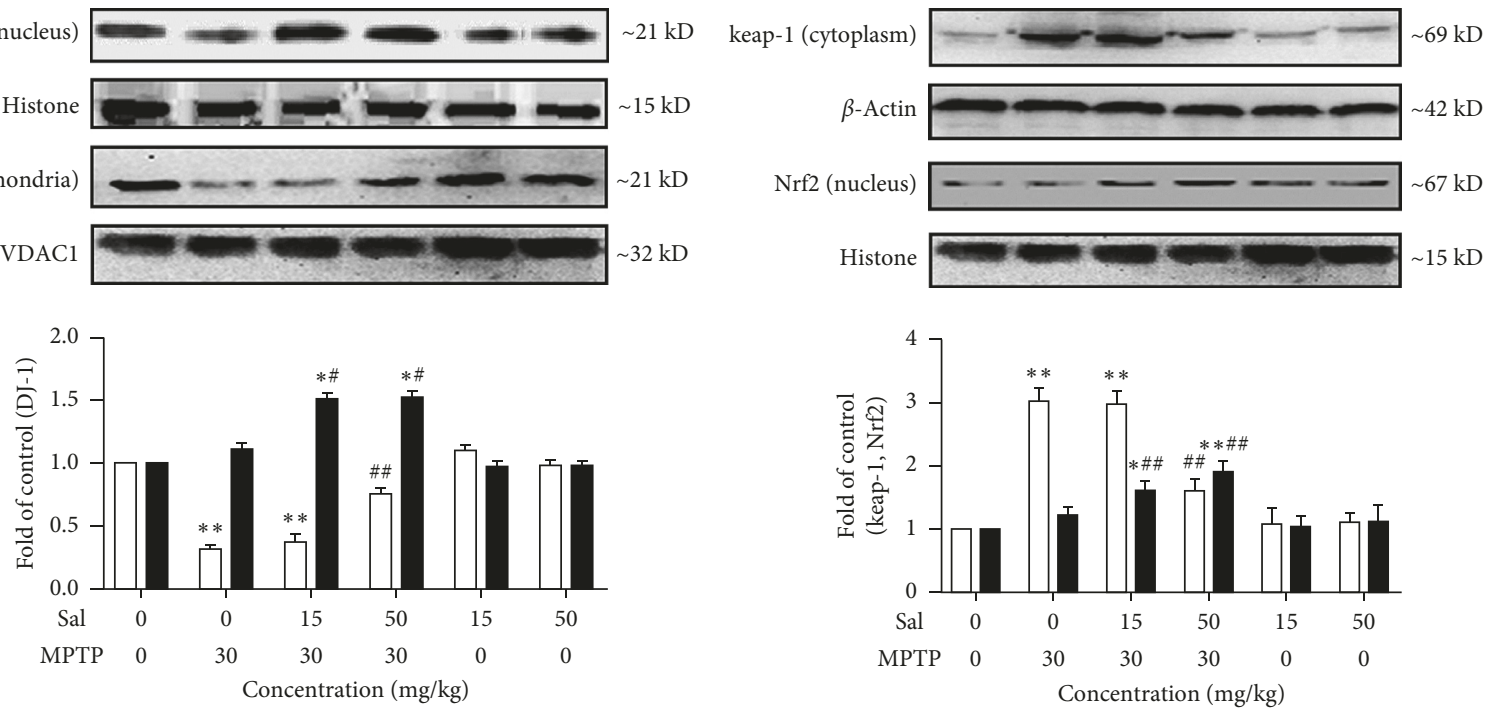

$\square$ DJ-1 in nucleus

- DJ-1 in mitochondria

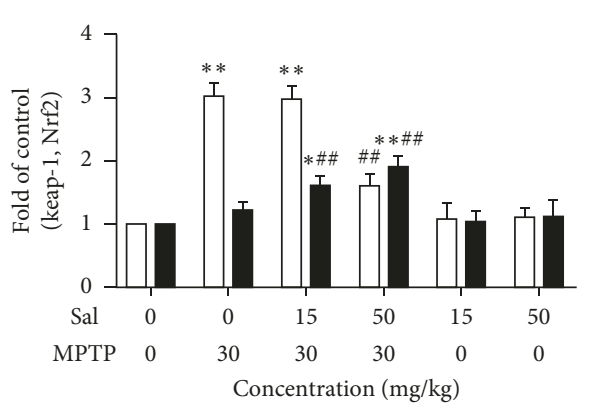

(c) 


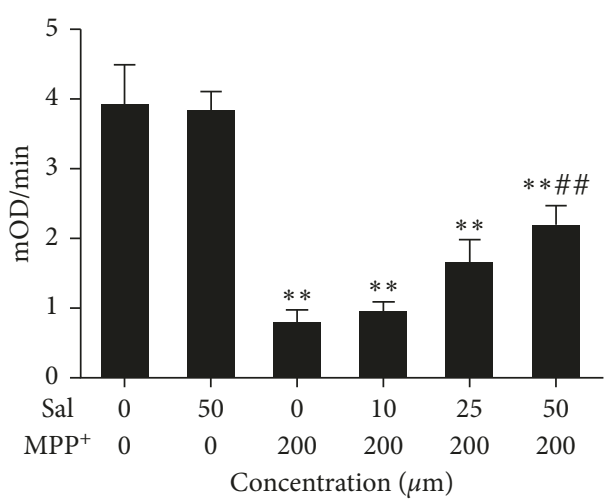

(a)

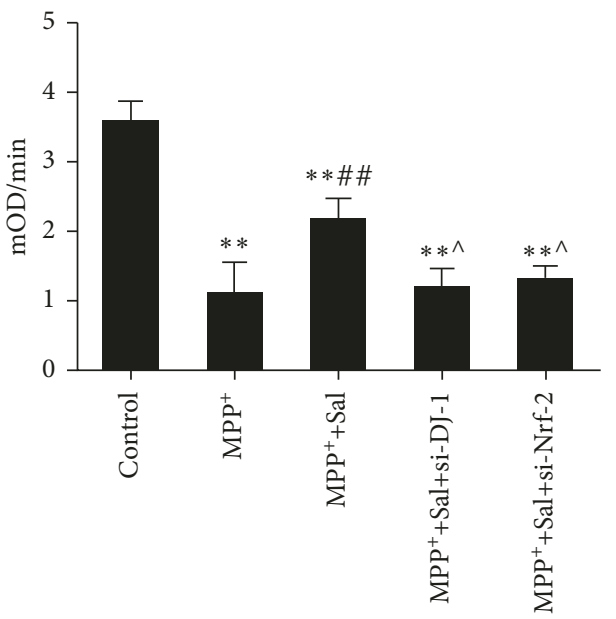

(c)

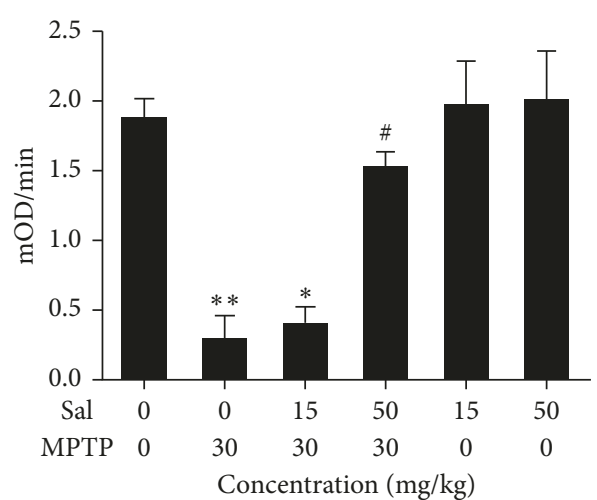

(b)

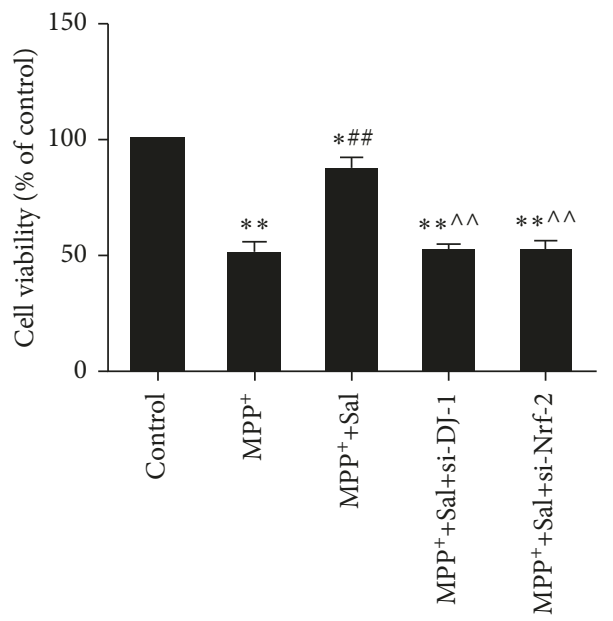

(d)

FiguRE 6: Role of DJ-1/Nrf2-mediated antioxidant effect for the maintenance of complex I activity and neuroprotective effect of Sal. Sal reversed $\mathrm{MPP}^{+} / \mathrm{MPTP}$-induced impairment of complex I activity in MN9D cells (a) and the substantia nigra of mice (b). Effect of silencing DJ-1 and Nrf2 on complex I activity (c) and cell viability (d) of MN9D cells. Each column represents the mean \pm SD $(n=3) .{ }^{*} P<0.05$ and ${ }^{* *} P<0.01$, compared with the control group; ${ }^{\#} P<0.05$ and ${ }^{\# \#} P<0.01$, compared with the $\mathrm{MPP}^{+} / \mathrm{MPTP}$-treated group; ${ }^{\wedge} P<0.05$ and ${ }^{\wedge} P<0.01$, compared with the $\mathrm{MPP}^{+}-$Sal treated group.

promoted the nuclear accumulation of DJ-1 and Nrf2 and the mitochondrial accumulation of DJ-1 and inhibited Keap1 expression, compared with the $\mathrm{MPP}^{+} / \mathrm{MPTP}$-treated group. To confirm the antioxidative stress effect of Sal was DJ-1/Nrf2-dependent, we silenced DJ-1/Nrf2 by using siRNA. Results showed silencing of DJ-1/Nrf2 inhibited the suppression of ROS generation and abrogated the nuclear/ mitochondrial accumulation of DJ-1 and the nuclear accumulation of Nrf2 induced by Sal.

Due to the fact that oxidative stress is intimately linked to complex I dysfunction, numerous neuroprotective mechanisms converge on preserving complex I via antioxidant therapeutic strategies $[31,32]$. We first investigated the effect of Sal on complex I, and results indicated that Sal effectively prevented the decrease in complex I activity against $\mathrm{MPP}^{+}$/ MPTP. To further investigate whether DJ-1/Nrf2-mediated antioxidative stress involved in the observed effect of Sal on maintenance of complex I activity and improvement of cell injury, we silenced DJ-1 and Nrf2 by using siRNA. Our present study demonstrated silencing of DJ-1 and Nrf2 abolished the increase in complex I activity and cell viability elicited by Sal. The above data supported that maintenance of complex I activity by Sal through DJ-1/Nrf2-mediated antioxidant pathway serves as a crucial mechanism by which Sal attenuates complex I dysfunction and plays neuroprotective roles in the $\mathrm{MPP}^{+} / \mathrm{MPTP}$-induced $\mathrm{PD}$ model.

In accordance with our previous studies, the findings support the idea that Sal exhibits potent neuroprotective effects against the $\mathrm{MPP}^{+} / \mathrm{MPTP}$-induced $\mathrm{PD}$ model through preserving complex I activity via DJ-1/Nrf2-mediated antioxidant pathway. The neuroprotective effect of Sal may hold promise as a preventive therapy of PD.

However, our study has limitations. It is noteworthy that the expression of Nrf2 is cell-type-specific. In contrast to neuronal cell lines, there are data showing that the neuronal $\mathrm{Nrf} 2$ activity is low in cortical neurons due to epigenetic repression of Nrf2 gene promoter early in development [33]. The results of Nrf2 presented in our study are restricted to cell lines and are not applicable to bona fide neurons. Therefore, further studies on bona fide neurons are needed to verify the neuroprotective effect of Sal. Another limitation of our study is that, in addition to the antioxidant pathway of 
Nrf2 in maintaining complex I activity, it also has roles in the regulation of mitochondrial biogenesis and mitophagy. Ahuja et al. found that, in Nrf2-KO MEFs and Nrf2-KO mice, mitochondrial biogenesis was decreased, as evidenced by the decrease in the content of mtDNA, the protein expression of ETC complexes, and the genes involved in replication and transcription of mtDNA [34]. Another important process that maintains mitochondrial quality control is mitophagy. PINK1 (encoded by PARK6 gene) interacts with Parkin (encoded with PARK2 gene) to maintain mitochondrial membrane integrity and target dysfunctional mitochondria for autophagy. There are data showing that Nrf2 can upregulate PINK1 expression, and exogenous Nrf2 stimulation protected PINK-KO and PINK1-WT cell lines from DA-induced toxicity $[35,36]$. Thus, more studies should be conducted to investigate whether Sal confers neuroprotective effects via regulating mitochondrial biogenesis and mitophagy.

\section{Data Availability}

The data used to support the findings of this study are available from the corresponding author upon request.

\section{Conflicts of Interest}

The authors declare that they have no conflicts of interest.

\section{Authors' Contributions}

Ruru Li and Songhai Wang have contributed equally to this study.

\section{Acknowledgments}

This work was supported by the National Nature Science Foundation of China (Grant number 81173590).

\section{Supplementary Materials}

Figure S1: the results of the DJ-1 and Nrf2 expression after transfecting siRNA for $72 \mathrm{~h}$. Si-Nrf2 (3\#, $25 \mathrm{nM})$ and si-DJ$1(3 \#, 100 \mathrm{nM})$ were used in the following experiments. Figure S2: silencing DJ-1 and Nrf2 abolished the inhibitory effect of Sal on ROS generation. Figure S3: silencing DJ-1 and Nrf2 inhibited Sal-induced nuclear/mitochondrial accumulation of DJ-1 and the nuclear accumulation of $\mathrm{Nrf} 2$. Figure S4: the gray value and quantitative analysis of the nuclear DJ-1 expression in MN9D cells. Figure S5: the gray value and quantitative analysis of the mitochondrial DJ-1 expression in MN9D cells. Figure S6: the gray value and quantitative analysis of the nuclear DJ-1 expression in the SN of mice. Figure S7: the gray value and quantitative analysis of the mitochondrial DJ-1 expression in the SN of mice. Figure S8: the gray value and quantitative analysis of the nuclear Nrf2 expression in MN9D cells. Figure S9: the gray value and quantitative analysis of the nuclear Nrf2 expression in the SN of mice. (Supplementary Materials)

\section{References}

[1] D. Ghezzi and M. Zeviani, "Assembly factors of human mitochondrial respiratory chain complexes: physiology and pathophysiology," Advances in Experimental Medicine and Biology, vol. 748, pp. 65-106, 2012.

[2] L. V. Kalia and A. E. Lang, "Parkinson's disease," The Lancet, vol. 386, no. 9996, pp. 896-912, 2015.

[3] H. Braak, K. D. Tredici, U. Rüb, R. A. I. de Vos, E. N. H. Jansen Steur, and E. Braak, "Staging of brain pathology related to sporadic Parkinson's disease," Neurobiology of Aging, vol. 24, no. 2, pp. 197-211, 2003.

[4] C. Henchcliffe and M. F. Beal, "Mitochondrial biology and oxidative stress in Parkinson disease pathogenesis," Nature Clinical Practice Neurology, vol. 4, no. 11, pp. 600-609, 2008.

[5] J. Zhao, S. Yu, Z. Yan et al., "Oxidative modification and its implications for the neurodegeneration of Parkinson's disease," Mol Neurobiol, vol. 54, pp. 1-15, 2016.

[6] H. Yokoyama, H. Kuroiwa, R. Yano, and T. Araki, "Targeting reactive oxygen species, reactive nitrogen species and inflammation in MPTP neurotoxicity and Parkinson's disease," Neurological Sciences, vol. 29, no. 5, pp. 293-301, 2008.

[7] T. Laszlo, S. Ildiko, and A. V. Vera, "Initiation of neuronal damage by complex I deficiency and oxidative stress in Parkinson's disease," Neurochemical Research, vol. 29, no. 3, pp. 569-577, 2004.

[8] A. Bose and M. F. Beal, "Mitochondrial dysfunction in Parkinson's disease," Journal of Neurochemistry, vol. 139, pp. 216-231, 2016.

[9] D. A. Johnson and J. A. Johnson, "Nrf2-a therapeutic target for the treatment of neurodegenerative diseases," Free Radical Biology and Medicine, vol. 88, pp. 253-267, 2015.

[10] H. Ariga, K. T. Niki, I. Kato et al., "Neuroprotective function of DJ-1 in Parkinson's disease," Oxidative Medicine \& Cellular Longevity, vol. 2013, Article ID 683920, p. 9, 2013.

[11] P. V. Raninga, G. Di Trapani, and K. F. Tonissen, "The multifaceted roles of DJ-1 as an antioxidant," Advances in Experimental Medicine and Biology, vol. 1037, pp. 67-87, 2017.

[12] H.-M. Chiang, H.-C. Chen, C.-S. Wu, P.-Y. Wu, and K.-C. Wen, "Rhodiola plants: chemistry and biological activity," Journal of Food and Drug Analysis, vol. 23, no. 3, pp. 359-369, 2015.

[13] P. Getova and M. Damianka, "Effects of Rhodiola rosea extract on passive avoidance tests in rats," Open Medicine, vol. 8, no. 2, pp. 176-181, 2013.

[14] W. Songhai, H. Hong, C. Lei et al., "Protective effects of salidroside in the MPTP/MPP(+)-induced model of Parkinson's disease through ROS-NO-related mitochondrion pathway," Molecular Neurobiology, vol. 51, no. 2, pp. 718-728, 2015.

[15] W. Zhang, H. He, H. Song et al., "Neuroprotective effects of salidroside in the MPTP mouse model of Parkinson's disease: involvement of the PI3K/Akt/GSK3 $\beta$ pathway," Parkinson's Disease, vol. 2016, Article ID 9450137, 9 pages, 2016.

[16] T.-T. Huang, D.-L. Hao, B.-N. Wu, L.-L. Mao, and J. Zhang, "Uric acid demonstrates neuroprotective effect on Parkinson's disease mice through Nrf2-ARE signaling pathway," Biochemical and Biophysical Research Communications, vol. 493, no. 4, pp. 1443-1449, 2017.

[17] H. Kuniishi, S. Ichisaka, M. Yamamoto et al., "Early deprivation increases high-leaning behavior, a novel anxiety-like behavior, in the open field test in rats," Neuroscience Research, vol. 123, article S0168010216303121, 2017. 
[18] L. Heredia, M. Torrente, M. T. Colomina, and J. L. Domingo, "Assessing anxiety in C57BL/6J mice: a pharmacological characterization of the open-field and light/dark tests," Journal of Pharmacological and Toxicological Methods, vol. 69, no. 2, pp. 108-114, 2014.

[19] A. P. Gureev, M. Y. Syromyatnikov, T. M. Gorbacheva, A. A. Starkov, and V. N. Popov, "Methylene blue improves sensorimotor phenotype and decreases anxiety in parallel with activating brain mitochondria biogenesis in mid-age mice," Neuroscience Research, vol. 113, pp. 19-27, 2016.

[20] E. B. Kurutas, "The importance of antioxidants which play the role in cellular response against oxidative/nitrosative stress: current state," Nutrition Journal, vol. 15, no. 1, p. 71, 2015.

[21] Z. Yi, V. L. Dawson, and T. M. Dawson, "Oxidative stress and genetics in the pathogenesis of Parkinson's disease," Neurobiology of Disease, vol. 7, no. 4, pp. 240-250, 2000.

[22] B. Damien, P. Paul-Antoine, and S. Alain, "Transcriptional mutagenesis induced by 8-oxoguanine in mammalian cells," PLoS Genetic, vol. 5, no. 7, article e1000577, 2009.

[23] F. Sesti, S. Liu, and S.-Q. Cai, "Oxidation of potassium channels by ROS: a general mechanism of aging and neurodegeneration?," Trends in Cell Biology, vol. 20, no. 1, pp. 45-51, 2010.

[24] I. Fridovich, "Superoxide dismutase," Encyclopedia of Biological Chemistry, vol. 44, pp. 352-354, 2013.

[25] J. Wu, M. Bao, D. Ge et al., "The expression of superoxide dismutase in mytilus coruscus under various stressors," Fish \& Shellfish Immunology, vol. 70, pp. 361-371, 2017.

[26] I. N. Zelko, T. J. Mariani, and R. J. Folz, "Superoxide dismutase multigene family: a comparison of the CuZn-SOD (SOD1), Mn-SOD (SOD2), and EC-SOD (SOD3) gene structures, evolution, and expression," Free Radical Biology and Medicine, vol. 33, no. 3, pp. 337-349, 2002.

[27] X. Zou, B. A. Ratti, J. G. O’Brien et al., "Manganese superoxide dismutase (SOD2): is there a center in the universe of mitochondrial redox signaling?," Journal of Bioenergetics and Biomembranes, vol. 49, no. 4, pp. 325-333, 2017.

[28] B. Jeff, L. Mahadevan, K. J. Thomas et al., "Formation of a stabilized cysteine sulfinic acid is critical for the mitochondrial function of the parkinsonism protein DJ-1," Journal of Biological Chemistry, vol. 284, no. 10, pp. 6476-6485, 2009.

[29] C. Casey M, M. Richard S, C. Brian J et al., "DJ-1, a cancerand Parkinson's disease-associated protein, stabilizes the antioxidant transcriptional master regulator Nrf2," Proceedings of the National Academy of Sciences, vol. 103, no. 41, pp. 15091-15096, 2006.

[30] D. Malhotra, R. Thimmulappa, A. Navas-Acien et al., "Decline in NRF2-regulated antioxidants in chronic obstructive pulmonary disease lungs due to loss of its positive regulator, DJ1," American Journal of Respiratory and Critical Care Medicine, vol. 178, no. 6, pp. 592-604, 2008.

[31] R. Perfeito, T. Cunha-Oliveira, and A. C. Rego, "Revisiting oxidative stress and mitochondrial dysfunction in the pathogenesis of Parkinson disease-resemblance to the effect of amphetamine drugs of abuse," Free Radical Biology and Medicine, vol. 53, no. 9, pp. 1791-1806, 2012.

[32] J. S. Park, R. L. Davis, and C. M. Sue, "Mitochondrial dysfunction in Parkinson's disease: new mechanistic insights and therapeutic perspectives," Current Neurology \& Neuroscience Reports, vol. 18, no. 5, p. 21, 2018.

[33] K. F. S. Bell, B. Al-Mubarak, M. Martel et al., "Neuronal development is promoted by weakened intrinsic antioxidant defences due to epigenetic repression of Nrf2," Nature Communications, vol. 6, no. 1, 2015.
[34] M. Ahuja, N. Ammal Kaidery, L. Yang et al., "Distinct Nrf2 signaling mechanisms of fumaric acid esters and their role in neuroprotection against 1-methyl-4-phenyl-1,2,3,6tetrahydropyridine-induced experimental Parkinson's-like disease," Journal of Neuroscience, vol. 36, no. 23, pp. 63326351, 2016.

[35] M. Hitoshi, T. Hitoshi, L. Sulai et al., "NRF2 regulates PINK1 expression under oxidative stress conditions," PloS One, vol. 10, no. 11, Article ID e0142438, 2015.

[36] A. T. Dinkova-Kostova, L. Baird, K. M. Holmström, C. J. Meyer, and A. Y. Abramov, "The spatiotemporal regulation of the Keap1-Nrf2 pathway and its importance in cellular bioenergetics," Biochemical Society Transactions, vol. 43, no. 4, pp. 602-610, 2015. 


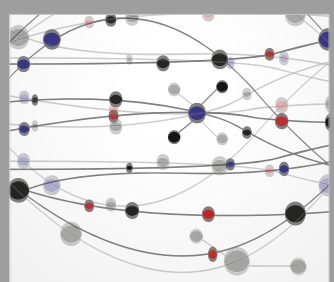

The Scientific World Journal
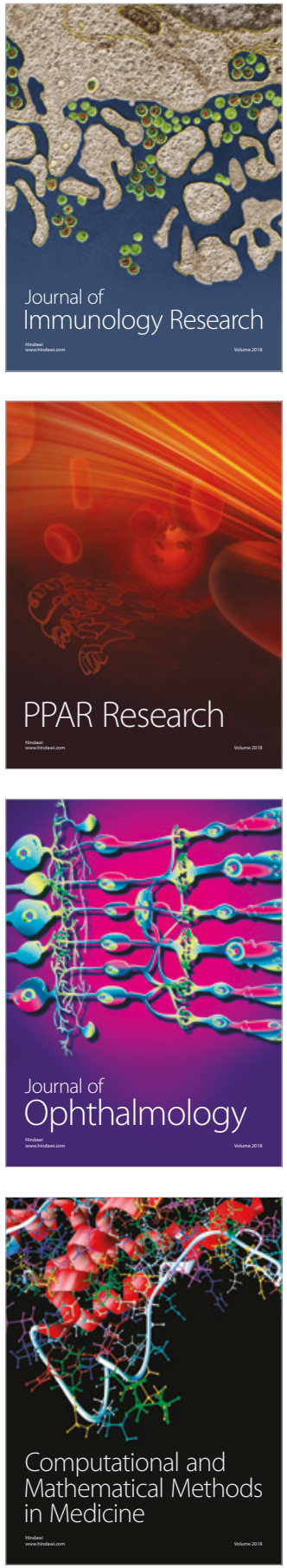

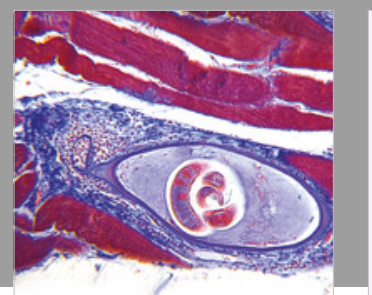

Gastroenterology Research and Practice

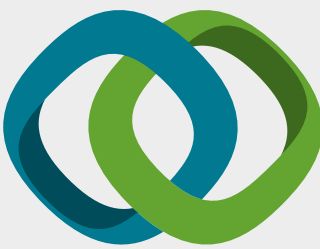

\section{Hindawi}

Submit your manuscripts at

www.hindawi.com
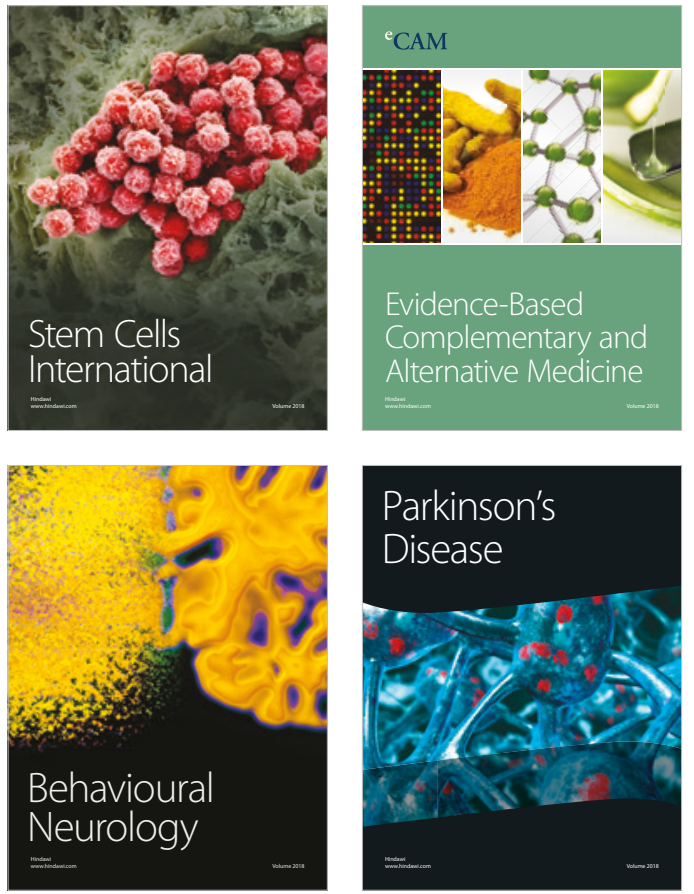

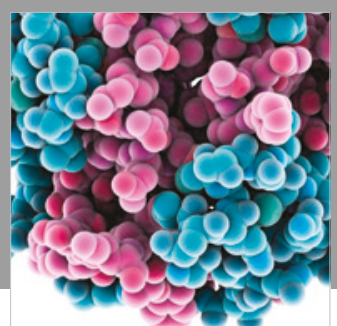

ournal of

Diabetes Research

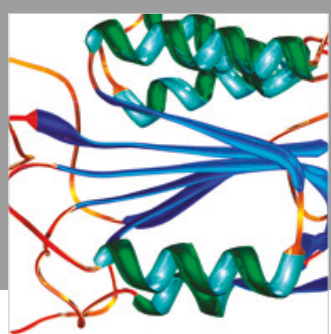

Disease Markers
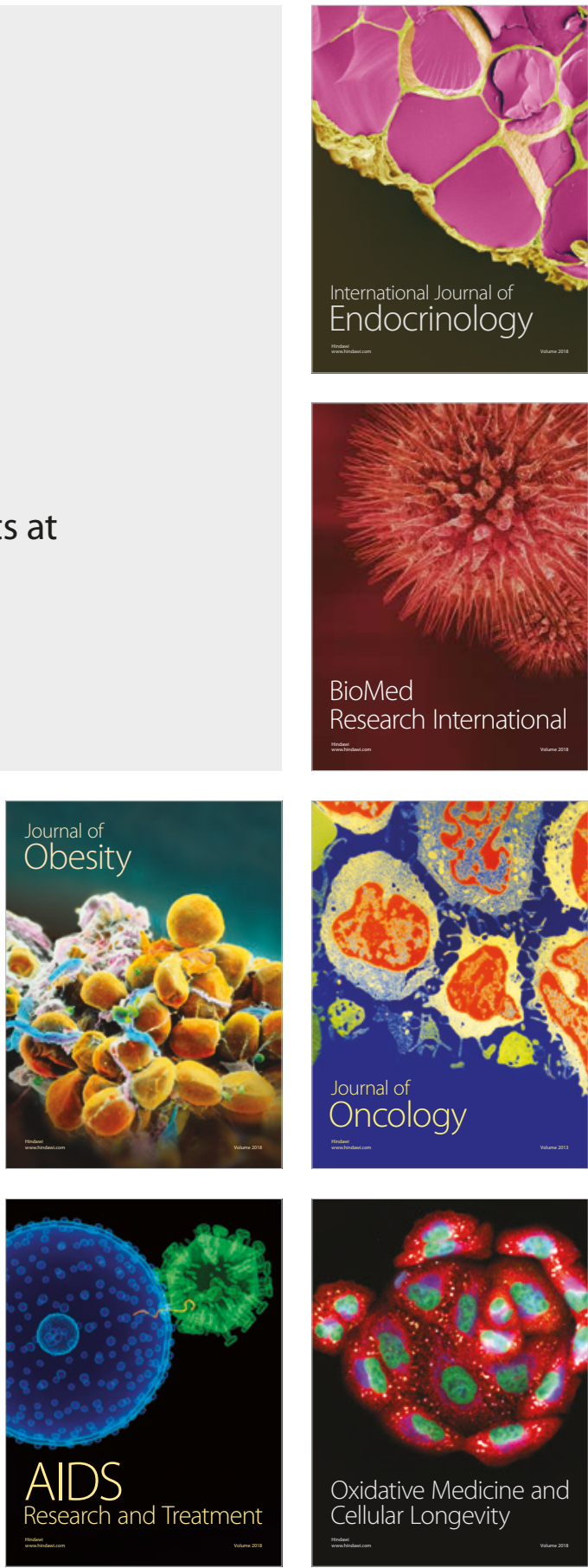